\title{
Integrated Behavioral Health for Preschool Children in Pediatric Primary Care
}

\author{
Michael W. Yogman, MD \\ Susan Betjemann, LICSW \\ Amelia Eppel, MEd \\ Nathan Yuen, BS
}

\begin{abstract}
Behavioral problems in preschool children have led to increased rates of expulsion and suspension from preschool. This case report describes the management of children aged 0-5 with behavioral concerns in a pediatric practice. Identified children were referred to an integrated behavioral health provider who provided accessible evaluation and treatment to parents and children, as well as consultation to childcare providers. The report describes case examples of how these services prevented adverse outcomes at the preschool age for three children.
\end{abstract}

\section{Introduction}

The epidemiology of pediatrics in the USA has shifted dramatically in the past twenty years; the top five disabilities affecting US children are now mental or behavioral health concerns rather than physical ones. ${ }^{1}$ In 2016, 1 out of 6 US children aged 2-8 suffered from a diagnosed behavioral, developmental, or mental health disorder, with boys being more likely than girls to have a diagnosed disorder. ${ }^{2,3}$ These issues include not only mental health disorders but also issues which are relational between children and caregivers. ${ }^{4}$ Behavioral health disorders interrupt children's education and often delay access to mental health services. ${ }^{5}$

Address correspondence to Michael W. Yogman, MD, Department of Pediatrics, Cambridge Hospital, Harvard Medical School, 14 Wyman Rd, Cambridge, MA 02138, USA. (mwyogman01@gmail.com)

Susan Betjemann, LICSW, Yogman Pediatric Associates, Cambridge, MA, USA.

Amelia Eppel, MEd, Department of Integrated Studies in Education, McGill University, Montreal, Canada.

Nathan Yuen, BS, Yale School of Nursing, Orange, CT, USA.

Journal of Behavioral Health Services \& Research, 2021 625-633. (C) 2021 National Council for Behavioral Health, corrected publication 2021. DOI 10.1007/s11414-021-09754-4 
The rates of preschool expulsion are high and have been linked to a lack of holistic support for teachers managing children with behavioral issues. ${ }^{6,7}$ Therefore, mental health services that function to support a child across the settings of home and school are crucial for preventing the fallout that occurs from under-achievement at the early stages of education. Wraparound mental health services have begun to develop in the field of early childhood. The state of Connecticut has put into place one of the first statewide programs, the Early Childhood Consultation Program (ECCP). With promotion, prevention, and intervention services for children with behavioral and mental health concerns, mitigation of suspension/expulsion from early care and education settings has been achieved. The ECCP pursues this aim through consultation, capacity building, and individualized treatment planning with families and schools. ${ }^{8}$ Two randomized trials of Connecticut's ECCP showed significant decreases in preschool teacher-rated challenging behaviors and a reduction in challenging behaviors by toddlers in infant/toddler childcare centers. ${ }^{9}, 10$

In 2015, the national childcare provider Head Start proposed rule changes severely limiting suspensions, prohibiting expulsions, and requiring programs to engage a mental health consultant in an effort to achieve this. ${ }^{11}$ Analysis of the effectiveness of this change has not yet been made; however, with leadership on this issue being taken at both the state and national levels, it is clear that mental health consultation for childcare providers and families is important for minimizing adverse early outcomes.

If children do not receive the behavioral healthcare that they need, they are more likely to underachieve academically. ${ }^{12}$ Recent studies have begun to find that most adult psychological problems have their roots in early childhood and have begun to investigate the high prevalence of emotional and behavioral problems that emerge in 3-5 year-olds. ${ }^{13}$ Community-based epidemiological studies show rates of psychiatric diagnoses in US children ranging from 13 to $27 \% .{ }^{14-16}$ Approximately $7.1 \%$ of children aged 3-17 have a diagnosed anxiety disorder, and $9.4 \%$ of children aged 2-17 have a diagnosis of ADHD, while 7.4\% have a diagnosed behavioral problem such as oppositional defiance disorder (ODD), or a conduct disorder. ${ }^{17}$ Perhaps most common, however, are behavioral issues and parental disciplinary problems that do not rise to a level requiring a DSM diagnosis but are nevertheless problematic to manage. More troubling data shows that only $25 \%$ of those identified with a behavioral or mental health issue receive treatment, and it is often inadequate in quality and quantity. ${ }^{18}$

Currently, the most common model of care for children with behavioral health disorders emphasizes referrals to outside behavioral health providers. There are multiple barriers to accessing outside care, including a lack of providers, long wait times, insufficient insurance coverage, and social stigma. ${ }^{19}$ Many providers who are in high demand do not accept insurance payment for their services, which further increases the barrier to care. ${ }^{20}$ Childcare providers dealing with disruptive child behavior have limited access to mental health consultation and support.

One way to improve access to behavioral healthcare and consultation for childcare providers is to integrate it into the pediatric primary care setting. The integrated model has been shown to be effective in adult primary care for improving quality outcomes and being cost-efficient. ${ }^{21}$ Studies have also shown that integration improves pediatric access to care and treatment outcomes for common behavioral health diagnoses. ${ }^{22}$

The stress of the coronavirus pandemic and the accompanying increase in economic and racial disparities has increased the need for early childhood mental health services. Further integration of behavioral health services into pediatric primary care settings can adapt interventions for particular pediatric populations. Studies have highlighted the value of "warm handoffs," effective collaboration with community programs (childcare, home visiting, early intervention) and focusing on parents as well as children in order to mitigate the impact of social determinants of health and further promote health equity. ${ }^{23-25}$ 
The following three case studies describe how the services provided by an integrated behavioral health provider located in a pediatric primary care practice were key in preventing adverse outcomes for preschool-age children. This was achieved through the evaluation and treatment of both parents and children, as well as consultation and care coordination with childcare providers. This model meets the need for child mental health consultation for childcare providers while supporting the family in a familiar setting.

\section{Methods}

This paper describes case studies from the subsample of preschool children from a quality improvement project that was conducted at a pediatric primary care office. ${ }^{26}$ This practice serves 2000 children who are predominantly middle income (only 5\% Medicaid) and young (25\% are under age 5). From August 2013 through December 2016, 78 preschool children were identified as having behavioral health concerns. These patients were identified by their pediatricians and referred to the practice LICSW for evaluation. ${ }^{27}$ Over 240 services were provided by the LICSW to 66 patients in the practice (42 males, 24 females). The reasons for referral included the following: disruptive behavior/ aggression (20), maternal depression (16), autism assessment and care coordination (16), attachment concerns (6), anxiety (3), divorce (3), and isolation (2). The age composition at referral was as follows: under age 1 (14), 1 year old (4), 2 years old (14), 3 years old (19), 4 years old (11), and 5 years old (4). Race/ethnicity was predominantly Caucasian (46), Asian (14), Black (5), and Hispanic (1). The number of preschool families seeking treatment rose from two in 2013 to over 25 in 2016. The twelve preschool patients not seen by the LICSW were managed by their pediatrician, often with LICSW consultation. Informed consent was obtained from all families in the study in coordination with an approved protocol by the Mt Auburn Hospital IRB.

The project - a behavioral health integration model-co-located a Licensed Clinical Social Worker (LICSW) trained in child behavioral healthcare into the practice, along with a parent partner/care coordinator. The LICSW has had extensive training in early childhood mental health, CBT techniques, and the Triple $\mathrm{P}$ parenting intervention ${ }^{28}$ and has provided consultation and treatment to patients with a focus on socio-emotional development and supporting families with young children. The Triple P program was extensively used to provide dual generation (parent and child) services. This parenting intervention aims to increase the knowledge, skills, and confidence of parents and to reduce the prevalence of mental health, emotional, and behavioral problems in children based on principles of community psychology. ${ }^{29}$ This project targets the goals of the Triple Aim: to improve patient experience, population health quality, and healthcare costs. ${ }^{30}$ By targeting preschool-age children, this project seeks to mitigate the issues of academic underachievement at the very start of a child's education.

Although the sample size was too small for formal analysis, some data on patient experience and quality health outcomes was collected, although unfortunately not on the three cases reported here. Three measures were used to evaluate patient experience. The Parenting Stress Index (PSI) ${ }^{31}$ was used before and after intervention to evaluate the impact on family stress levels. The PSI is a survey given to parents to assess the level of stress they are experiencing with their child. A percentile score is calculated. The perception of quality of care received was evaluated after treatment using the FamilyCentered Care Assessment for Families (FCCA-F) ${ }^{32}$ and the Client Satisfaction Questionnaire (CSQ8). ${ }^{33}$ The FCCA consists of 24 questions scored 1 to 5 , with 5 being the most positive. The total score ranges from 24 to 120 . The CSQ has 8 questions scored from 1 to 4 . Higher scores indicate greater satisfaction with care. Quality behavioral health outcomes were evaluated using the Baby and Preschool Pediatric Symptom Checklist (PPSC/BPSC). ${ }^{34}$ 


\section{Results}

\section{Patient experience}

Data collection on parent experience of stress (PSI) was collected from 12 families. Out of the 12 families, only 6 families completed the post-intervention survey. The mean stress percentile score for these 6 families decreased from the 74th to the 34th percentile. Five families completed the post-intervention FCCA-F survey. The average total score was 90.6 out of 120 . Four families completed the post-intervention CSQ-8 survey. Overall satisfaction was high; 3 of 4 patients scored above $90 \%$ with an average score of $89 \%$.

\section{Quality outcome}

Eight patients completed BPSC scores before and after intervention, 5 of them improved. The three that did not improve were all males. Five patients completed the PPSC before and after the intervention. Only two improved and both were females. This data suggests that female preschool children were more responsive to the short-term intervention.

\section{Case studies}

\section{Case 1: Jay}

Jay is a $3 \frac{1}{2}$-year-old African American male who lives with his mother, father, and 2-year-old brother. Family history is notable for bipolar disorder and substance abuse in his father and anxiety and depression in his mother. Jay was a healthy newborn except for mild jaundice. His early history was notable for an umbilical hernia, eczema, headaches, and delayed language. Subsequently, evidence of seizures was found on an EEG. He was receiving early intervention services for delayed language but was not felt to meet the criteria for autism. His mother works overnight shifts and his father works full time. Jay's family qualifies for Head Start and Jay currently attends the full-day program. He receives speech therapy once a week at Head Start for a General Language Delay. No other services are provided at this time.

Chief complaint Jay is in danger of being expelled from his Head Start program due to his disruptive and aggressive behaviors in the classroom. His mother reached out to Jay's PCP for assistance and recommendations. The initial call to the PCP was made on December 12th, and the referral to the office social worker was December 13th and an appointment for the family with the office social worker was scheduled for the same day.

Intervention The social worker met with Jay and his mother for an urgent appointment. His mother shares concerns that Jay's classroom is loud and chaotic which may trigger his behavioral outbursts. Jay's mother recognizes that his behavior is disruptive and aggressive and agrees to pursue any available behavioral health supports that Head Start can offer. She is, however, unable to bring Jay to outside appointments due to work schedule constraints. Jay's mother agrees to allow the social worker to contact the Head Start staff to advocate for further evaluation and services. The social worker also demonstrates some relaxation techniques for Jay to try to identify possible coping techniques.

Care coordination Multiple conversations were had between the LICSW and Head Start personnel, as well as the behavioral health clinic contracted by Head Start and the family. In total, 
three phone calls were made to the education coordinator at Head Start to discuss their concerns and to provide insight and consultation. The education coordinator agreed that Jay should move classrooms and agreed to help the family request an evaluation from the Behavioral Health Clinic as well as an evaluation from the public school system.

Outcome By February, Jay had moved to another classroom with more experienced teachers. He also began individual therapy with a clinician assigned to his Head Start program. Jay was assessed by the public school system and met the requirements for a preschool placement with an Individualized Education Plan (IEP) for speech and occupational therapy. Jay's mother reported being pleased with the outcome and felt that his behavior had calmed significantly.

Follow-up Jay's mother called back the following September. Jay had started at his new school but his behavior had regressed and the school was now stating that he needed to be moved to another site for more intensive services. Jay's mother also confided in the social worker that there is a history of domestic violence between her and his father and that she and the children had recently moved out to stay with extended family. The social worker met with Jay's mother to assess for safety concerns and to direct her to any needed resources. With all of the changes of moving and starting a new school for Jay, his mother was worried that another transition to a new school would be too difficult. Jay's mother agreed to let the social worker provide consultation to the school and advocate for him to remain at the school. Over the next 2 months, the social worker called the parent 13 times, met with her twice, called the therapist 5 times, and spoke with the school 5 times. The social worker advocated for better coordination of support and services at school and for a classroom placement that would better address Jay's needs. The social worker and school counselor worked together to formulate a plan to better support Jay and his academic and social/emotional needs. Jay was moved to a class with a more experienced teacher and his speech, OT, and behavior supports were coordinated with the classroom. The school also created a behavior support plan to identify goals and necessary supports. His mother reported that Jay's behavior improved over this time as did the communication between the family and his school and therapists.

\section{Case 2: Carl}

Carl is a 4-year-old Caucasian male who lives with his mother, father, and 2.5-year-old brother. Carl's father works full-time and is often traveling while his mother stays home with the children. The family is originally from Greece and lived in Germany for many years. The family is in the USA for the next 3 years for Carl's father's work. The parents speak Greek and German at home and are both fluent in English. Carl and his brother speak Greek and German with a few English phrases. The family is heavily involved in the Greek church and community in their neighborhood. Carl attends the preschool attached to the family's Greek Church full time (5 days/week). Carl has not been assessed for any additional behavioral health services.

Chief complaint Carl's mother called his PCP for assistance as the preschool is struggling to address Carl's frequent behavioral outbursts at school. The school has reported that he was aggressive with peers and staff and had a very difficult time transitioning from activity to activity. Carl's mother stated she is looking for guidance and resource information. The initial call to the PCP was made on November 6th. The referral to the social worker was made on November 7th and the social worker was in contact with Carl's mother that same day. 
Intervention Carl's mother talked with the social worker by phone and shared her concerns about Carl's aggressive behaviors at home and at school. She also asked the social worker to get in touch with his preschool as they have asked to have Carl seen by a behavioral health clinician. The social worker made contact with the preschool by phone and they requested that the clinician visit onsite and observe Carl at the preschool. This observation was scheduled for November 20th. Appropriate permission and releases were obtained prior to the scheduled observation. Carl was observed playing with peers, participating in music class, and transitioning between several activities. Carl demonstrated some rigidity and frustration in his play with peers and seemed best able to interact with peers when he had close support and guidance from a staff member. After the observation, the social worker met with the preschool staff including the director, classroom teacher, and education coordinator. The staff were worried about Carl and were looking for advice on how to best support him at preschool. The preschool staff and social worker discussed methods of supporting Carl during the day, paying special attention to extra support during transitions. The staff and social worker explored possible interventions including using a quiet space to help with emotional regulation and using fidget/squishy tools to help relieve pressure and frustration during the day. The social worker met with Carl's mother to follow-up on the preschool meeting, providing her with a copy of her notes and suggestions from the meeting. They also discussed her concerns about Carl's behavior at home. Carl's mother expressed interest in the Triple P program, an evidencebased short-term clinical intervention focused on parental support and psychoeducation for families whose children are exhibiting challenging behavior. Carl's mother attended six sessions of Triple P with a clinician and kept in communication with Carl's preschool about the tools she was using at home to try and create consistency between the two environments.

Outcome Carl's mother's verbal reports and data sheets recorded measurable behavioral improvement for Carl at home and at school. She also shared that he is less defiant and was motivated by positive reinforcement used at home. Carl's mother reported feeling better equipped to address his challenging behaviors by setting limits and communicating clearly with him. Together with the social worker, Carl's mother followed up with the preschool which was able to implement some of the strategies that the social worker suggested, with moderate success.

Follow-up Carl's mother reported continued behavioral improvements at his next well-child visit. She has been better able to communicate with the preschool about how to best intervene and support Carl. The preschool continues to work on their programmatic supports for Carl and reports some areas of success. Carl is scheduled to transition out of the Greek Church preschool and will begin attending public school in the fall.

\section{Case 3: David}

David is a 5-year-old male that lives with his mother, father, and 17-month-old brother. Both parents are employed by a local university and David attends a preschool affiliated with this institution. David has not been assessed for any outside services. David has recently experienced disruption as his previous preschool closed suddenly and he was then cared for by his grandmother for several weeks before transitioning to his current school.

Chief complaint David's mother called the office social worker about concerns shared by David's preschool. The school was struggling to address his behavioral outbursts which seem to occur daily and have involved physical aggression. His mother was looking for parenting guidance and 
intervention as well as consultation with David's preschool. The initial call to the social worker was made on April 18th and an immediate phone consultation was had with the parent. An inoffice appointment was scheduled for April 26th for David and both his parents.

Intervention The social worker spoke with David's mother over the phone and discussed current behavioral concerns at school. David's behavior at home had improved somewhat over time but he struggled with emotional regulation, transitions, and listening to directions. At the meeting with David and both his parents, the social worker reviewed triggers, challenging behaviors, and possible coping skills. David's parents provided permission for the social worker to speak with the preschool's education coordinator who had been working with David in the classroom around his behavior. The social worker spoke with the education coordinator 3 times by phone and continued to meet with the parents and David in person, as well as communicating through the practice's patient portal. The education coordinator provided some insight into David's potential triggers from her observations, which were used to inform intervention approaches. The social worker and education coordinator discussed a potential behavior support plan that provided David with a space to take a break from the classroom if he is overstimulated. A system of positive reinforcement for expected (regulated) behaviors was also put into place. The social worker focused on exploring and practicing coping skills in sessions with David and his parents, to be used in the classroom as well as at home.

Outcome Both parents and the education coordinator reported a decrease in physical aggression and a moderate increase in appropriate use of coping skills during transitions at school and at home. David's parents, the social worker, and the education coordinator remained in close contact to monitor progress and make adjustments to the plan as needed. The education coordinator reported improvements in behavior and is working with staff to better identify David's triggers to help prepare him to use his coping skills in advance. The parents reported feeling satisfied with the engagement and communication between providers. The social worker continued to support and work with the family. They have applied to their public school special education department for a formal evaluation in the hope that he will be eligible for services with an IEP, which may help David access social and emotional supports as he transitions to public school in the fall.

Follow-up This case is ongoing, and the social worker remains a support for the family around behavioral challenges. The family will be transitioning again for the summer, traveling to another country. The parents have enrolled David in a nature camp ahead of this transition where he seems to be doing well and reports feeling excited about his upcoming travels. His parents will continue to practice coping skills with David while traveling and will continue to use positive reinforcement to encourage regulated behaviors.

\section{Discussion and Implications for Behavioral Health}

Previous reports have documented the benefits of integrated behavioral health in primary care. ${ }^{33}$, 34, 26 These case reports illustrate the opportunity for coordinated behavioral healthcare intervention during the preschool years, addressing problems early before they become serious emotional disorders and consulting with preschools to prevent expulsion. While these case studies focus on short-term behavioral improvement for these children, the interventions provided to young children at risk are likely to have long-term benefits for ongoing academic and social success. Furthermore, parenting interventions can have impact well beyond the initial issue. 
Pediatricians are now routinely screening for developmental delays, autism, behavioral concerns, and parental postpartum mood disorders. When screens are positive, the easy availability in the pediatric office of a behavioral health provider prevents long wait times for referrals and the stigma of seeing a consultant outside of the regular medical setting. Additionally, a rapid therapeutic alliance develops because of the established trusted relationship between pediatrician and parent, which enables a transfer of trust to the behavioral health provider. Using the Triple P Primary Care treatment model, the behavioral health provider has been able to provide support to families struggling with their children's challenging behaviors. These challenging behaviors may be severe enough to put the child at risk of losing their daycare or preschool place.

The easy and timely access to a behavioral health provider with early childhood expertise exemplified in these case studies illustrates the benefit of a "warm handoff." In many cases, after a warm handoff from the pediatrician, using the combined approach of an intervention with the family and consultation with daycare or preschool providers, the behavioral health provider is able to help the family, child, and childcare providers find a shared method of addressing challenging behaviors and preventing the child's expulsion from preschool. Although onsite observations in a childcare center are not considered billable services, the observation and evaluation of children in these settings can be included in billing for evaluation. Parent support groups run by the behavioral health provider in the pediatric office in the first month of life decrease the social isolation many parents experience at this time and provide an environment in which the discussion of early childhood behavioral concerns is encouraged.

Given the cost and challenge of providing mental health services to older children with serious disorders, this model of integrated care provides a pathway for pediatricians to provide earlier intervention and primary prevention of childhood behavioral concerns. Unfortunately, the behavioral health services described in this report were not adequately covered by fee for service billing but only as a result of independent grant support. While data suggests that this model may produce long-term healthcare cost savings, insurance companies have yet to make this team model of the medical home financially sustainable.

Funding Funding was provided by the Harvard Pilgrim Quality Grant Foundation.

\section{Declarations}

Conflict of Interest The authors declare no competing interests.

\section{References}

1. Slomski A. Chronic mental health issues in children now loom larger than physical problems. Journal of American Medical Association. 2012; 308(3): 223.

2. Torio CM, Encinosa N, Berdahl T, et al. Annual report on health care for children and youth in the United States: national estimates of cost, utilization and expenditures for children with mental health conditions. Academic Pediatrics. 2015; 15(1): 19-35.

3. Cree RA, Bitsko RH, Robinson LR, et al. Health care, family, and community factors associated with mental, behavioral, and developmental disorders and poverty among children aged 2-8 years - United States, 2016. Morbidity \& Mortality Weekly Report. 2018; 67(5):1377-1383.

4. Ghandour RM, Sherman LJ, Vladutiu CJ, et al. Prevalence and treatment of depression, anxiety, and conduct problems in U.S. children. The Journal of Pediatrics. 2019; 206: 256-267.

5. Pascoe J, Wood D, Duffey T, et al. Mediation and adverse effects of child poverty in the US. Pediatrics. 2016; 137. doi:e2016/0340 
6. Gilliam WS. Implementing policies to reduce the likelihood of preschool expulsion. Foundation for Child Development Policy Series Brief Series. No. 7. New York, NY: Foundation for Child Development; 2008. http://www.ziglercenter.yale.edu/publications/ PreKExpulsionBrief2_tcm350-34772_tcm350-284-32.pdf.

7. Gilliam WS, Shahar G. Prekindergarten expulsion and suspension: rates and predictors in one state. Infants and Young Children. 2006; 19: $228-245$.

8. About Us. Early Childhood Consultation Partnership (ECCP). http://www.eccpct.com/About/. Accessed September 6, 2019.

9. Gilliam WS. Early Childhood Consultation Partnership: Results of Yale University's three statewide random-controlled evaluations. Final report with executive summary. New Haven, CT: Health Affairs; December 2014.

10. Gilliam W, Maupin A, Reyes C, et al. Do early educator's implicit biases regarding sex and race relate to behavior expectations and recommendations of preschool expulsions and suspensions? Presented at: US Administration for Children and Families 2016 State and Territory Administration Meeting, Alexandria, VA, September 28, 2016.

11. Head Start Performance Standards notice of proposed rulemaking [Section 1302.17: Suspension and Expulsion]. Head Start. https:// eclkc.ohs.acf.hhs.gov/policy/45-cfr-chap-XIII/1302-17-suspension-expulsion. Published November 2016.

12. Merikangas KR, Ames M, Cui L, et al. The impact of comorbidity of mental and physical conditions on the role of disability in the US adult household population. Archives of General Psychiatry. 2007; 64: 1180-1188.

13. Gleason MM, Goldson E, Yogman MW. Addressing early childhood emotional and behavioral problems. Pediatrics. 2016;138(6). doi:e2016/3025

14. Bufferd SJ, Dougherty LR, Carlson GA, et al. Parent-reported mental health in preschoolers: findings using a diagnostic interview. Comprehensive Psychiatry. 2011; 52: 359-369. doi:https://doi.org/10.1080/15374416.2012.759225

15. Wichstrom L, Berg-Nielsen TS, Angold A, et al. Prevalence of psychiatric disorders in preschoolers. Journal of Child Psychology and Psychiatry. 2012; 53: 695-705. doi:https://doi.org/10.1111/j.1469-7610.2011.02514.x

16. Dougherty LR, Leppert KA, Merwin SM, et al. Advances and directions in preschool mental health research. Child Development Perspectives. 2015; 0(0): 1-6. doi:https://doi.org/10.1111/cdep12099

17. Danielson ML, Bitsko RH, Ghandour RM, et al. Prevalence of parent-reported ADHD diagnosis and associated treatment among U.S. children and adolescents, 2016. Journal of Clinical Child and Adolescent Psychology. 2018; 47(2): 199-212.

18. Jensen PS, Goldman E, Offord D, et al. Overlooked and underserved: "Action signs" for identifying children with unmet mental health needs. Pediatrics. 2011; 128: 970-979. doi:https://doi.org/10.1542/peds.2009-0367

19. Benzer JK, Beehler S, Miller C, et al. Grounded theory of barriers and facilitators to mandated implementation of mental health care in the primary care setting. Depression Research and Treatment. 2012; 1-11.

20. Bishop TF, Press MJ, Keyhani S, et al. Acceptance of insurance by psychiatrists and the implications for access to mental health care. Journal of American Medical Association Psychiatry. 2014; 71(2): 176.

21. Kates N, Anderson E. Canadian approach to integrated care. In Feinstein R, Connelly J, Feinstein M. Integrating Behavioral Health and Primary Care. Oxford, UK: Oxford University Press; 2017. doi: https:/doi.org/10.1093/med/9780190276201.001.0001

22. Reiss-Brennan B, Brunisholz KD, Dredge C, et al. Association of integrated team-based care with health care quality, utilization and cost. Journal of American Medical Association. 2016; 316(8): 826-834.

23. McAuliffe M, Riley AR. Introduction to the special issue on integrated pediatric primary care. Clinical Practice in Pediatric Psychology. 2020; 8(3): 211-216.

24. Young ND, Matthews BL, Pan AY, et al. Warm handoff or cold shoulder? Clinical Practice in Pediatric Psychology. 2020; 8(3): 241246.

25. McCabe MA, Leslie L, Counts N, et al. Pediatric integrated care as the foundation for healthy development across the life span. Clinical Practice in Pediatric Psychology. 2020: 8(3): 278-287.

26. Yogman MW, Betjemann S, Sagaser A, Brecher L. Integrated behavioral health care in pediatric primary care: a quality improvement project. Clinical Pediatrics. 2018; 57(4): 461-470.

27. Sanders MR. Triple P-positive parenting program as a public health approach to strengthening parenting. Journal of Family Psychology. 2008; 22(3): 506-517.

28. Berwick DM, Nolan TW, Whittington J. The triple aim: care, health and cost. Health Affairs (Millwood). 2008; 27: 759-769.

29. Abidin RR. Parenting Stress Index. $4^{\text {th }}$ ed. Lutz, FL: Psychological Assessment Resources; 2012.

30. Wells N, Bronheim S, Zyzanski S, et al. Psychometric evaluation of a consumer- developed family-centered care assessment tool. Maternal Child Health Journal. 2015; 19:1899-1909.

31. Larsen DL, Attkisson CC, Hargreaves WA, et al. Assessment of client/ patient satisfaction: development of a general scale. Evaluation Program Planning. 1979; 2: 197-207.

32. Sheldrick RC, Hensson BS, Neger EN, et al. The Baby and Pediatric Symptom Checklist: development and initial validation of a new social/ emotional screening instrument for very young children. Academic Pediatrics. 2013; 13: 72-80.

33. Kolko DJ, Perrin E. The integration of behavioral health interventions in children's health care: services, science and suggestions. Journal Clinical Child Adolescent Psychology. 2014; 43: 216-228.

34. Kolko DJ. The effectiveness of integrated care on pediatric behavioral health: outcomes and opportunities. Journal of American Medical Association Pediatrics. 2015; 169: 894-896.

Publisher's Note Springer Nature remains neutral with regard to jurisdictional claims in published maps and institutional affiliations. 